\title{
Diagrama de Voronoi nas Métricas Euclidiana e do Táxi: Uma Exploração em GeoGebra
}

\section{Voronoi Diagram in Euclidian and Taxicab Metric: An Exploration Using GeoGebra}

\author{
Paula Roberta Scaburi dos Santos \\ Secretaria de Estado da Educação, Santa Catarina \\ prof.paulascaburi@gmail.com \\ Andres David Baez-Sanchez \\ Universidade Tecnológica Federal do Paraná, Curitiba \\ adavidbaez@gmail.com
}

\begin{abstract}
Resumo: Este trabalho apresenta uma exploração do conceito de diagrama de Voronoi na métrica Euclidiana e na métrica do táxi com a ajuda do ambiente de geometria dinâmica GeoGebra. São descritas visualizações dinâmicas para alguns resultados teóricos relacionados ao diagrama de Voronoi na métrica Euclidiana, assim como ilustrações dos conceitos de táxi-circunferência e táxi-mediatriz. Finalmente é implementado um procedimento para a representação das regiões de influência do diagrama de Voronoi na métrica do táxi.
\end{abstract}

Palavras-chave: diagrama de Voronoi; métrica Euclidiana; métrica do táxi; GeoGebra.

\begin{abstract}
This work presents an exploration of the Voronoi diagram in Euclidean and Taxicab metrics using the dynamic geometry environment GeoGebra. Using dynamic visualizations, some theoretical results related to Voronoi diagram in Euclidean metric are illustrated as well as the concepts of taxi-circumference and taxicab-bisector. Finally, a procedure is implemented to represent the regions of influence of the Voronoi diagram in the taxicab metric.
\end{abstract}

Key words: Voronoi diagram; Euclidian metric; táxicab metric; GeoGebra.

\section{Introdução}

A noção de diagrama de Voronoi de um conjunto de pontos $P$, pode ser relacionada à divisão do plano em um conjunto de regiões de influência, cada uma determinada pela proximidade entre os pontos da região e os pontos do conjunto $P$. As seguintes definições formalizam esta ideia:

Definição 1 Dado um conjunto $P=\left\{p_{1}, p_{2}, \ldots, p_{n}\right\}$ de pontos e uma métrica $d$ em $\mathbb{R}^{2}$, a célula de Voronoi $V_{d}\left(p_{i}\right)$ é definida como

$$
V_{d}\left(p_{i}\right)=\left\{q \in \mathbb{R}^{2}: d\left(q, p_{i}\right) \leq d\left(q, p_{j}\right) \text { para } j=1, \ldots, n\right\} .
$$

\begin{tabular}{llllll}
\hline RECEN & $19(2)$ & p.114-133 & jul/dez & 2017 & DOI:10.5935/RECEN.2017.02.01 \\
\hline
\end{tabular}


Caso não haja lugar a equívocos em relação à métrica, será usada a notação $V\left(p_{i}\right)$ no lugar de $V_{d}\left(p_{i}\right)$. A seguinte definição introduz o conceito de diagrama de Voronoi de um conjunto de pontos.

Definição $2 O$ diagrama de Voronoi de $P=\left\{p_{1}, p_{2}, \ldots, p_{n}\right\}$, denotado por $\operatorname{Vor}(P)$ é definido como a união das fronteiras de todas as células de Voronoi $V\left(p_{i}\right)$ correspondentes aos pontos do conjunto $P$.

Assim, cada célula de Voronoi $V\left(p_{i}\right)$ é o conjunto dos pontos que estão mais próximos ou à mesma distância de $p_{i}$ do que de qualquer outro ponto de $P$ e o diagrama de Voronoi $\operatorname{Vor}(P)$ é a união das fronteiras de todas as células de Voronoi. As Figuras 1 e 2 ilustram estes conceitos considerando a métrica euclidiana.

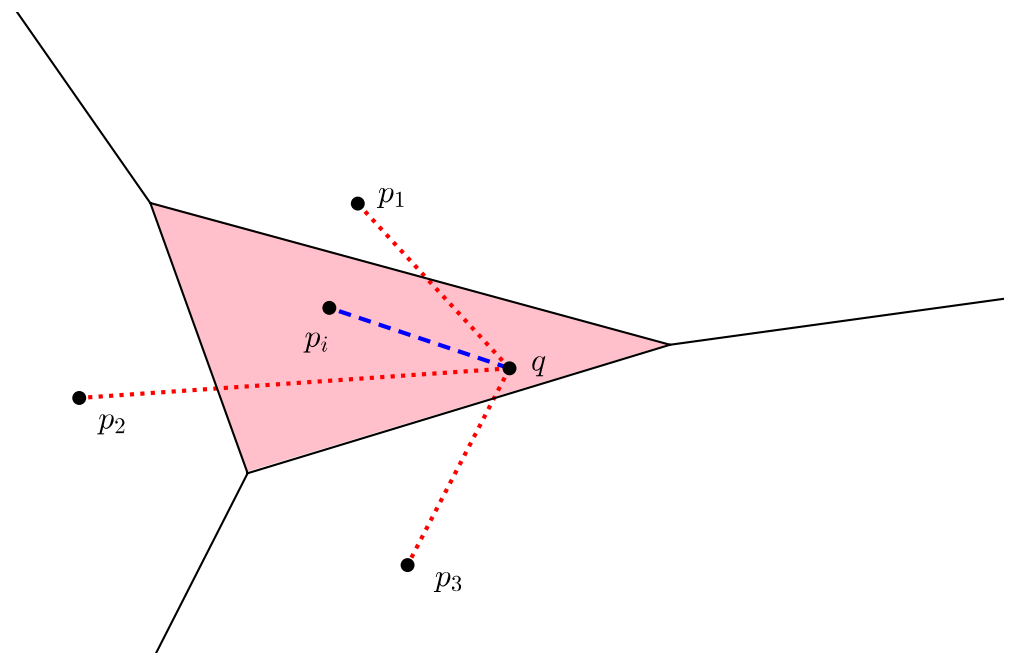

Figura 1. Célula de Voronoi $V\left(p_{i}\right)$

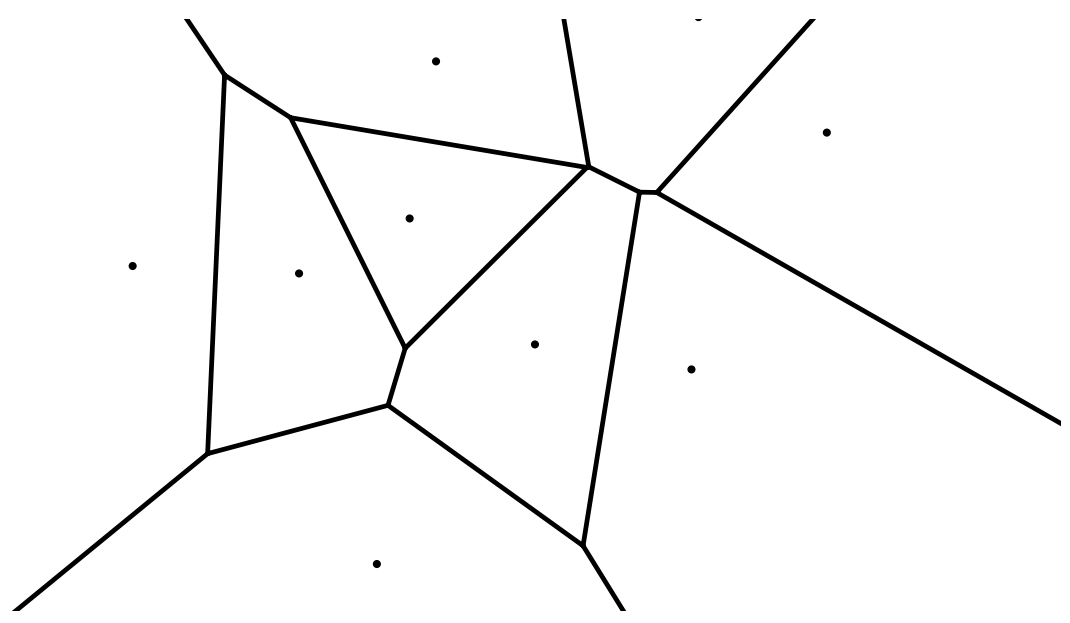

Figura 2. Diagrama de Voronoi $\operatorname{Vor}(P)$

É possível encontrar na literatura definições diferentes das aqui apresentadas. Em [1] por exemplo, o diagrama de Voronoi é definido como o conjunto das células e não como a união 
Revista Ciências Exatas e Naturais, Vol.19, nº.2, Jul/Dez, 2017

das fronteiras. Em [2] as células são definidas usando a desigualdade estrita $d\left(q, p_{i}\right)<d\left(q, p_{j}\right)$ no lugar da desigualdade $d\left(q, p_{i}\right) \leq d\left(q, p_{j}\right)$. Neste trabalho as definições apresentadas seguem as consideradas em [3].

Segundo [4], as primeiras apresentações formais do conceito do diagrama de Voronoi aparecem em trabalhos de Peter Gustav Lejeune Direchlet (1805-1859) e George Fedoseevich Voronoy (ou Voronoi) (1868-1908). Em 1854, o médico britânico John Snow utilizou uma ideia similar ao diagrama de Voronoi, para concluir que a maioria das pessoas que morreram na epidemia de cólera em Soho, distrito de Londres, moravam mais perto da bomba de água de Broad Street do que de qualquer outra bomba, mostrando assim a relação entre a água consumida e o surto da doença. Este fato é considerado como o início da epidemiologia moderna [5].

Estruturas relacionadas ao diagrama de Voronoi aparecem em várias áreas do conhecimento: astronomia, arqueologia, planejamento urbano, física, fisiologia, estudo de epidemias, ecologia, entre outras áreas [1]. O diagrama de Voronoi também é estudado na área de geometria computacional [3].

Este trabalho considera o uso do ambiente de geometria dinâmica GeoGebra para a exploração do conceito de diagrama de Voronoi na métrica Euclidiana e na métrica do táxi. Serão descritas algumas construções dinâmicas para ilustrar resultados teóricos relacionados ao diagrama na métrica Euclidiana, assim como visualizações dos conceitos de táxi-circunferência e táxi-mediatriz. Finalmente será considerado um procedimento para a representação das regiões de influência do diagrama de Voronoi na métrica do táxi.

\section{Diagrama de Voronoi na métrica Euclidiana}

A determinação explícita do diagrama de Voronoi na métrica Euclidiana, pode ser feita usando alguns dos vários algorítmos descritos na literatura $[1,2,3]$ mas quando for considerado um conjunto com um, dois ou três pontos, é possível determinar o diagrama de Voronoi de forma simples.

\subsection{Diagrama de Voronoi para um, dois ou três pontos}

Quando houver apenas um ponto, a célula de Voronoi será o plano todo e o diagrama de Voronoi será vazio. Para dois pontos $A$ e $B$, basta construir a mediatriz do segmento $\overline{A B}$. Sobre a mediatriz de $\overline{A B}$ estão os pontos que ficam a mesma distância dos dois pontos e as células de Voronoi são justamente os semiplanos fechados definidos pela mediatriz. O diagrama de Voronoi correspondente resulta ser a própria mediatriz do segmento formado pelos dois pontos.

Para três pontos, tem-se dois casos possíveis: pontos colineares ou não colineares. Considere primeiro o caso do diagrama de Voronoi para três pontos $A, B$, e $C$ não colineares. Inicialmente são determinadas as mediatrizes dos segmentos $\overline{A B}, \overline{B C}$ e $\overline{C A}$ como ilustrado na Figura 3. 


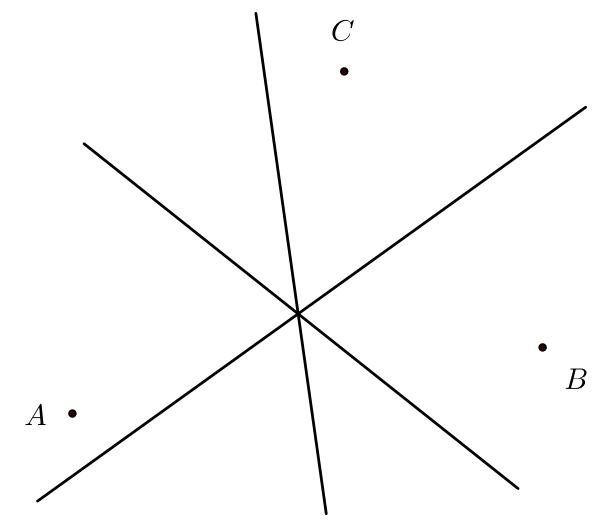

Figura 3. Construção das mediatrizes.

O ponto de encontro das mediatrizes é o ponto que está a mesma distância dos pontos A, $\mathrm{B}$ e $\mathrm{C}$ e será chamado de vértice do diagrama de Voronoi. Note que este ponto é justamente o circuncentro do triângulo $A B C$. Para determinar como serão as células de Voronoi para cada ponto, note que sobre a mediatriz do segmento formado por dois pontos, há pontos que estão mais próximos do terceiro ponto do que dos dois pontos que formam o segmento, assim esses pontos pertencem à região de influência do terceiro ponto. Basta eliminar então esses pontos em cada mediatriz para obter a representação adequada das células de Voronoi. Note, por exemplo, que para o ponto de influência $A$ na Figura 3 , a parte inferior à esquerda da mediatriz do segmento $\overline{B C}$ será desconsiderada, pois estes pontos estão mais próximos do ponto $A$ e portanto pertencem a região de influência de $A$. O mesmo será feito com as outra mediatrizes conforme a Figura 4:

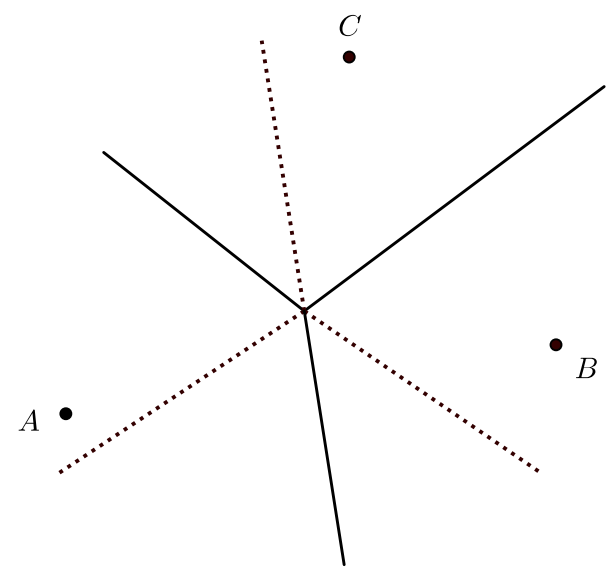

Figura 4. Eliminação dos segmentos que invadem as regiões de influência.

A figura restante, após a eliminação dos segmentos, será o diagrama de Voronoi para estes três pontos no plano. 
Revista Ciências Exatas e Naturais, Vol.19, nº.2, Jul/Dez, 2017

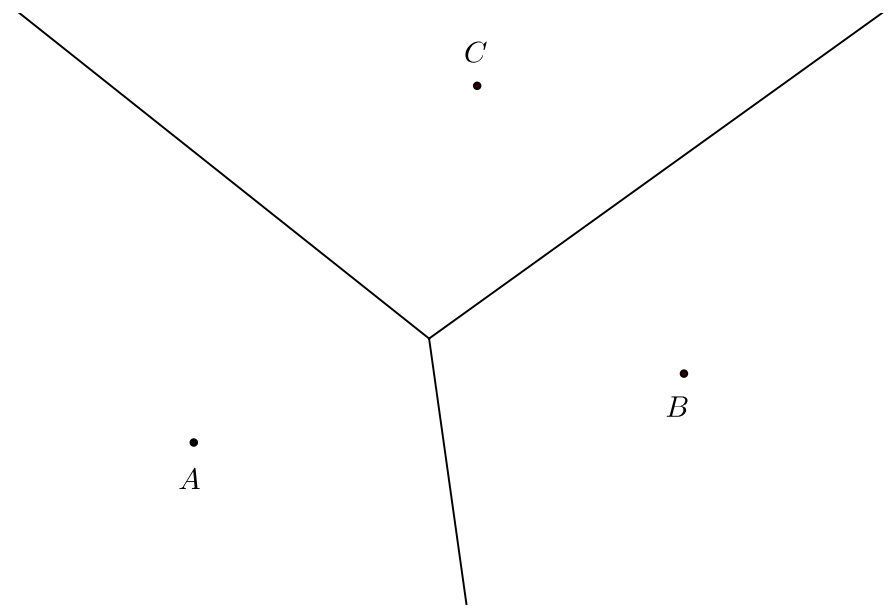

Figura 5. Diagrama de Voronoi para os pontos não colineares $A, B$ e $C$.

Se os três pontos forem colineares, o diagrama de Voronoi será formado por retas (mediatrizes) paralelas. Isto será enunciado e ilustrado no Teorema 3.

Todas as figuras consideradas na discussão anterior foram geradas usando o GeoGebra, mas é possível usar este software para uma exploração mais dinâmica de outras propriedades do diagrama de Voronoi.

\subsection{Usando o GeoGebra para explorar o diagrama de Voronoi na métrica Euclidiana}

O diagrama de Voronoi na métrica Euclidiana pode ser gerado no GeoGebra usando os comandos DiagramaDeVoronoi ou Voronoi como é ilustrado na Figura 6.

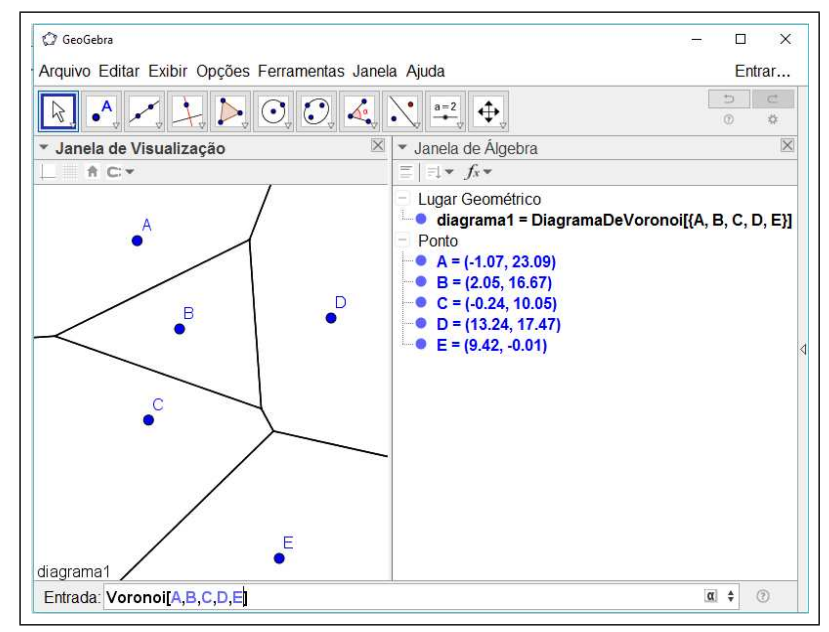

Figura 6. Diagrama de Voronoi na métrica Euclidiana no GeoGebra.

A seguir serão exploradas algumas propriedades do diagrama de Voronoi na métrica Euclidiana. As demonstrações das propriedades consideradas podem ser encontradas em [3] 
ou $[6]$.

Teorema 3 Seja $P=\left\{p_{1}, p_{2}, \ldots, p_{n}\right\}$ um conjunto de pontos no plano. Se todos os pontos forem colineares o Vor $(P)$ consiste em $n-1$ linhas paralelas. Caso contrário, $\operatorname{Vor}(P)$ é formado por segmentos de reta ou semirretas.

Este resultado pode ser ilustrado com facilidade no GeoGebra manipulando diretamente os pontos considerados e o comando Voronoi ou com ajuda de um controle deslizante.

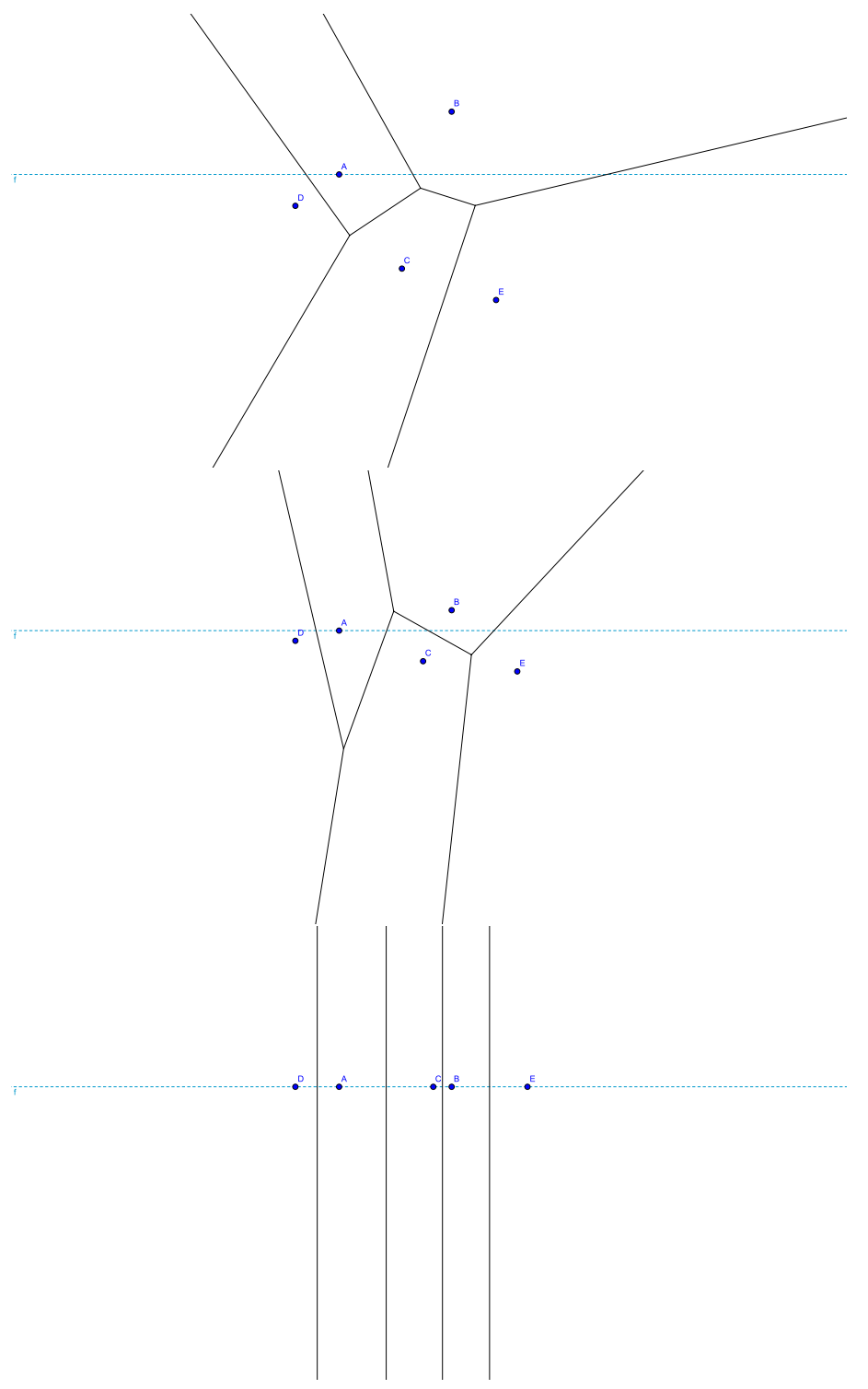

Figura 7. Diagrama de Voronoi: Pontos Não Colineares e Colineares. 
Revista Ciências Exatas e Naturais, Vol.19, nº.2, Jul/Dez, 2017

Na Figura 7, note como quanto mais próximos os pontos encontram-se da reta $f$, os segmentos de reta no diagrama de Voronoi vão mudando sua inclinação até se tornarem retas paralelas. Uma construção dinâmica correspondente pode ser acessada em https://ggbm.at/c9E3hgjG.

Os próximos três teoremas permitem determinar, através de circunferências, se um ponto qualquer do plano é ponto interior de alguma célula, está sobre uma única aresta, ou é um dos vértices do diagrama de Voronoi de um conjunto de pontos $P=\left\{p_{1}, p_{2}, \ldots, p_{n}\right\}$. Para estabelecer a classificação, dado um ponto $p \notin P$ será usada a notação $C_{p}$ para denotar um círculo com centro $p$ que não tem nenhum dos pontos do conjunto $P$ no seu interior.

Teorema 4 Um ponto $p$ é um ponto interior de $V\left(p_{i}\right)$ se e somente se existe um círculo $C_{p}$ tal que $p_{i}$ é o único ponto de $P$ na fronteira de $C_{p}$.

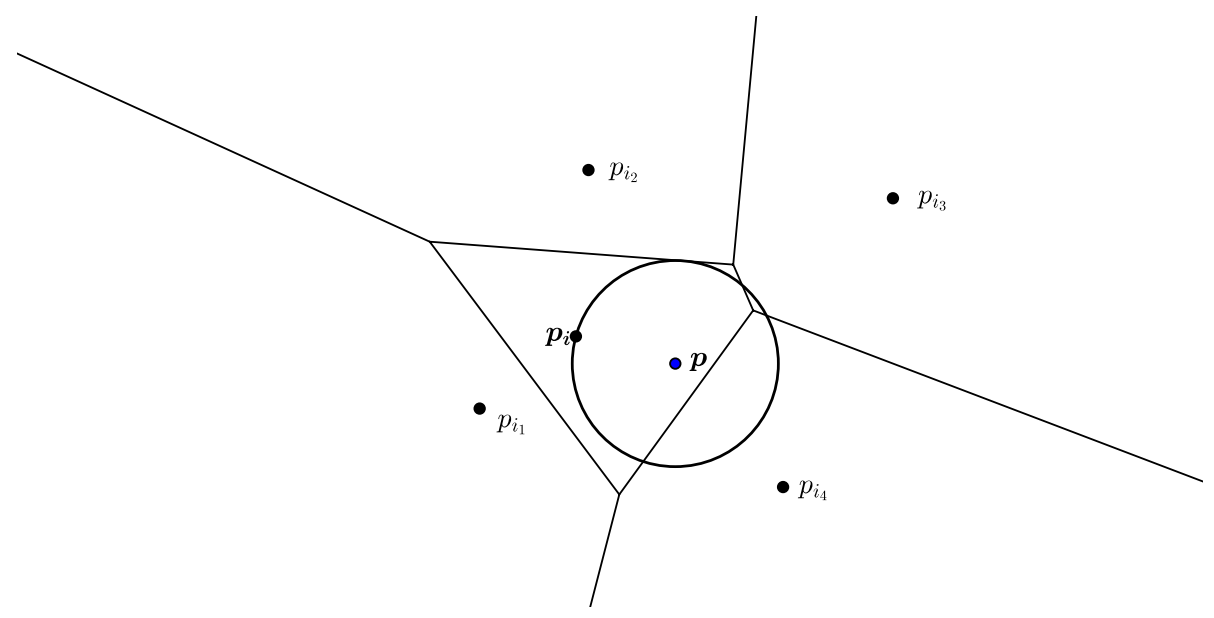

Figura 8. Ponto interior a $p_{i}$.

Note na Figura 8, que a circunferência em destaque, é a única com centro em $p$, que possui um ponto de $P$ na fronteira e nenhum ponto de $P$ no interior.

O Teorema 5 carateriza os pontos que pertencem exatamente a duas células de Voronoi, isto é, são pontos no interior relativo a uma das arestas e não são vértices.

Teorema 5 Um ponto $p$ é um ponto interior da aresta $V\left(p_{i}\right) \cap V\left(p_{j}\right)$ se, e somente se, existe um círculo $C_{p}$ tal que exatamente os pontos $p_{i}$ e $p_{j}$ de $P$, estão na fronteira de $C_{p}$.

Na Figura 9, é ilustrado o fato de que para um ponto $p$ no interior da aresta é possível construir um círculo $C_{p}$ com exatamente os pontos $p_{i}$ e $p_{j}$ na fronteira de $C_{p}$. A figura também ilustra o fato de que esta mesma propriedade é satisfeita por pontos $p^{\prime}$ e $p^{\prime \prime}$ sobre a aresta que estejam suficientemente próximos de $p$. 


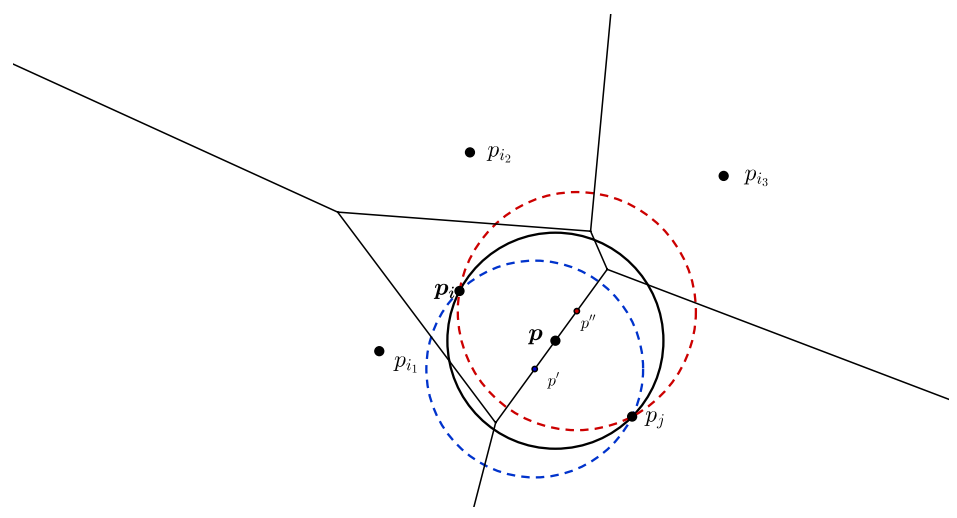

Figura 9. Pontos interiores a aresta de Voronoi.

Teorema 6 Um ponto $p$ é um vértice de Voronoi se, e somente se existe um círculo $C_{p}$ tal que há pelo menos três pontos de $P$ na fronteira de $C_{p}$.

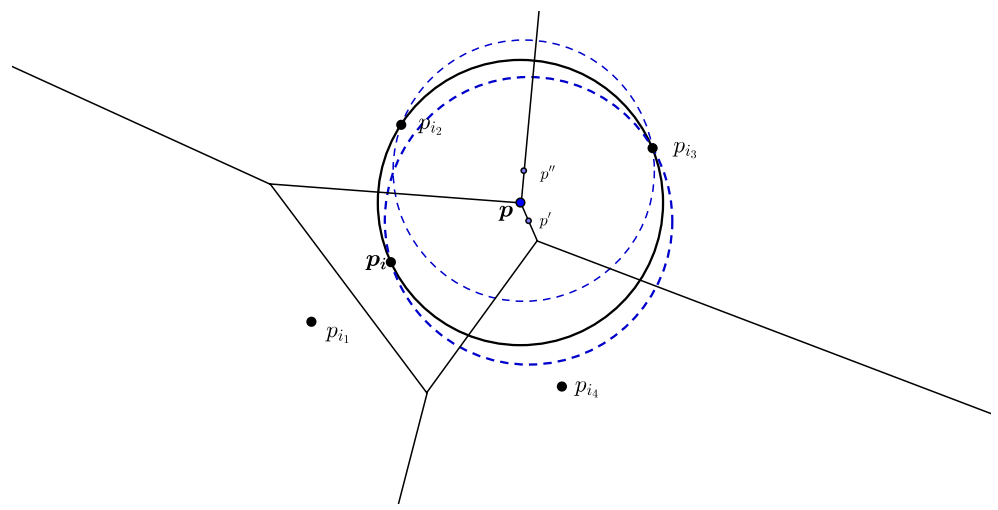

Figura 10. Ponto sobre o vértice de Voronoi.

Na Figura 10, é ilustrado o fato de que para um ponto $p$ que seja vértice do diagrama é possível construir um círculo $C_{p}$ com três ou mais pontos de $P$ na fronteira. Note que os pontos $p^{\prime}$ e $p^{\prime \prime}$ próximos de $p$, não têm esta propriedade.

Além de usar o GeoGebra para a construção de gráficos, é possível utilizar as suas ferramentas para ilustrar dinamicamente os três teoremas anteriores simultaneamente. Um exemplo de uma construção dinâmica simples neste sentido pode ser encontrada em https://ggbm. at/JpnqZnPr.

O seguinte resultado, carateriza as células de Voronoi em termos do fecho convexo do conjunto $P$.

Teorema 7 A célula de Voronoi $V\left(p_{i}\right)$ é ilimitada se, e somente se, $p_{i}$ está na fronteira do fecho convexo de $P$.

A demonstração deste resultado utiliza os teoremas anteriores de caraterização de pontos via circunferências [3]. É possível utilizar ferramentas básicas do GeoGebra para ilustrar diversas partes da demonstração [6] ou construir uma ilustração dinâmica do resul- 
Revista Ciências Exatas e Naturais, Vol.19, nº.2, Jul/Dez, 2017

tado. Um exemplo de uma construção dinâmica neste sentido pode ser encontrada em https://ggbm.at/PdBm5BvQ.

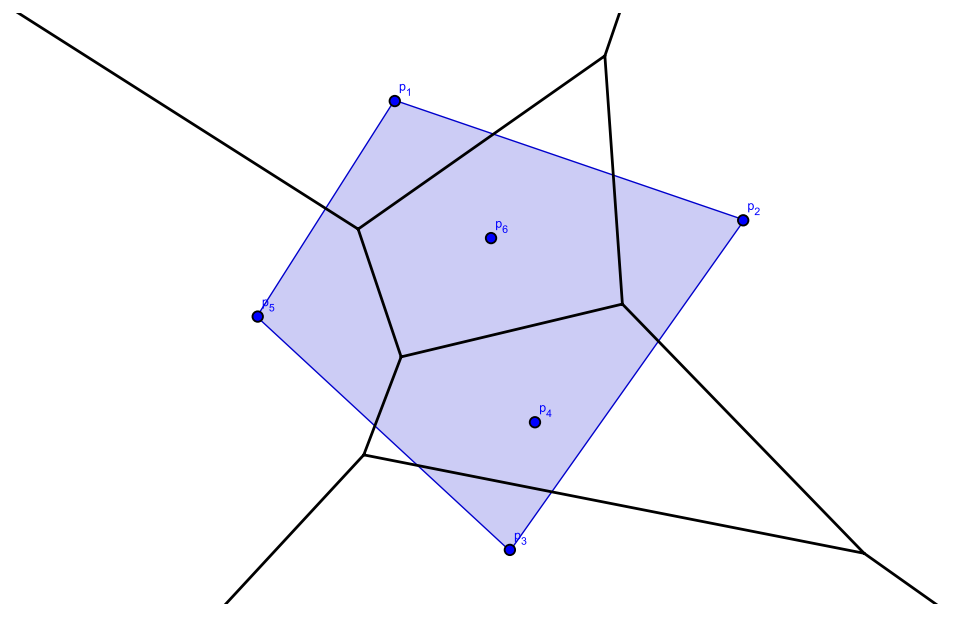

Figura 11. Células de Voronoi e Fecho Convexo de $P$.

\section{Métrica do Táxi}

Nesta seção será considerada a métrica do táxi. A métrica ou distância do táxi (também conhecida como métrica de Manhattan ou métrica $\left.L_{1}\right) d_{T}$ entre dois pontos $A=\left(x_{a}, y_{a}\right)$ e $B=\left(x_{b}, y_{b}\right)$ é definida como

$$
d_{T}(A, B)=\left|x_{a}-x_{b}\right|+\left|y_{a}-y_{b}\right|
$$

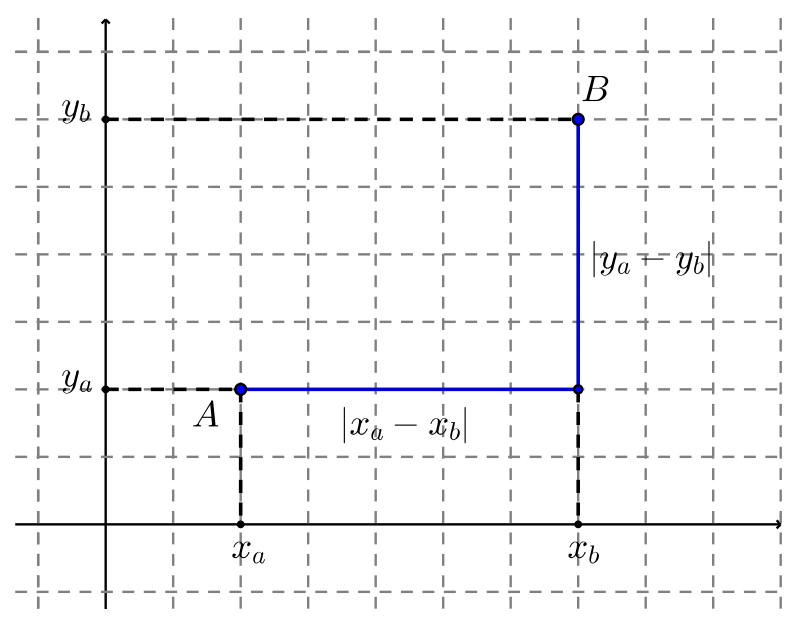

Figura 12. Distância ou métrica do táxi.

O nome de métrica do táxi é associado a esta métrica, pois numa região com ruas sempre paralelas ou ortogonais entre si, a distância total percorrida para ir de táxi do ponto $A$ ao 
SANTOS, P. S. e BAEZ-SANCHEZ, A. D.

ponto $B$ é igual a soma das distâncias percorridas em deslocamentos horizontais mais a soma das distâncias percorridas em deslocamentos verticais. Mas no plano, esta soma é justamente a soma definida na Equação 1 como é ilustrado na Figura 12. Nosso objetivo final é utilizar o GeoGebra para visualizar o diagrama de Voronoi de um conjunto de pontos na métrica do táxi, mas como não existe uma ferramenta pronta em GeoGebra para este propósito, na seção seguinte será desenvolvido um procedimento de representação aproximado. Para entender adequadamente este procedimento, nesta seção serão considerados os conceitos correspondentes à circunferência e mediatriz entre dois pontos na métrica do táxi e com a ajuda do GeoGebra, serão exploradas algumas das suas características. Mais detalhes dos conceitos e demonstrações dos resultados considerados ao longo desta seção podem ser encontrados em $[6,8]$.

\subsection{Táxi-Circunferência}

A táxi-circunferência de centro $C$ e raio $r$ é o lugar geométrico dos pontos no plano que na métrica do táxi distam $r$ unidades do ponto $C=\left(x_{c}, y_{c}\right)$. Assim, uma táxi-circunferência de raio $r$ e centro $C=\left(x_{c}, y_{c}\right)$ é formada pelos pontos $(x, y)$ que solucionam a equação:

$$
\left|x-x_{c}\right|+\left|y-y_{c}\right|=r .
$$

A seguinte proposição caracteriza este lugar geométrico.

Proposição 8 A táxi-circunferência de centro $C=\left(x_{c}, y_{c}\right)$ e raio $r$ é o quadrilátero de vértices $\left(x_{c}, y_{c}+r\right),\left(x_{c}+r, y_{c}\right),\left(x_{c}, y_{c}-r\right) e\left(x_{c}-r, y_{c}\right)$.

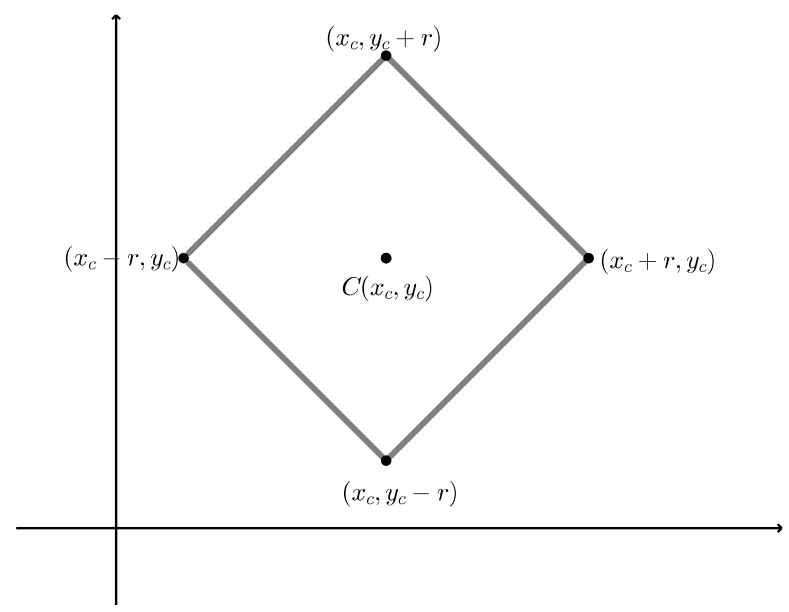

Figura 13. Táxi-circunferência.

A representação gráfica de uma táxi-circunferência também pode ser obtida no GeoGebra utilizando a expressão 2 como é ilustrado na Figura 14 
Revista Ciências Exatas e Naturais, Vol.19, nº.2, Jul/Dez, 2017

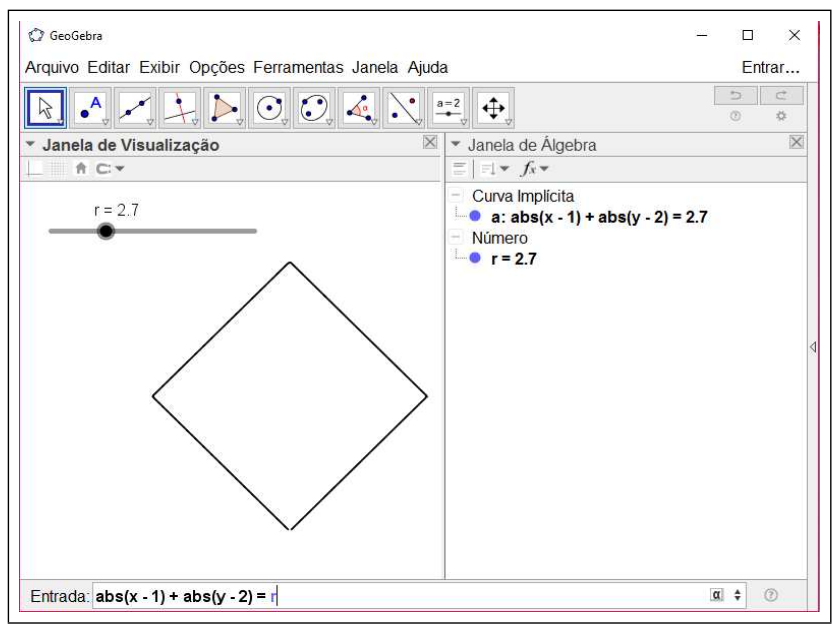

Figura 14. Táxi-circunferência no GeoGebra.

\subsection{Táxi-mediatriz}

A mediatriz entre os pontos $A$ e $B$ (ou mediatriz do segmento $\overline{A B}$ ) é definida como o lugar geométrico dos pontos $P$ tais que $d(P, A)$ é igual a $d(P, B)$. Considerando a definição da métrica do Táxi, para $P=(x, y), A=\left(x_{a}, y_{a}\right)$ e $B=\left(x_{b}, y_{b}\right)$ temos que $P$ pertence à táxi-mediatriz de $\overline{A B}$ se, e somente se

$$
\left|x-x_{a}\right|+\left|y-y_{a}\right|=\left|x-x_{b}\right|+\left|y-y_{b}\right| .
$$

Em princípio, parece natural usar diretamente a expressão anterior para gerar no GeoGebra a táxi-mediatriz entre dois pontos, no entanto, veremos que esta implementação direta pode produzir uma representação um tanto confusa em certos casos. Nas Figuras 15 e 16 é ilustrado o uso direto da expressão 3 para gerar a representação da táxi-mediatriz em GeoGebra.

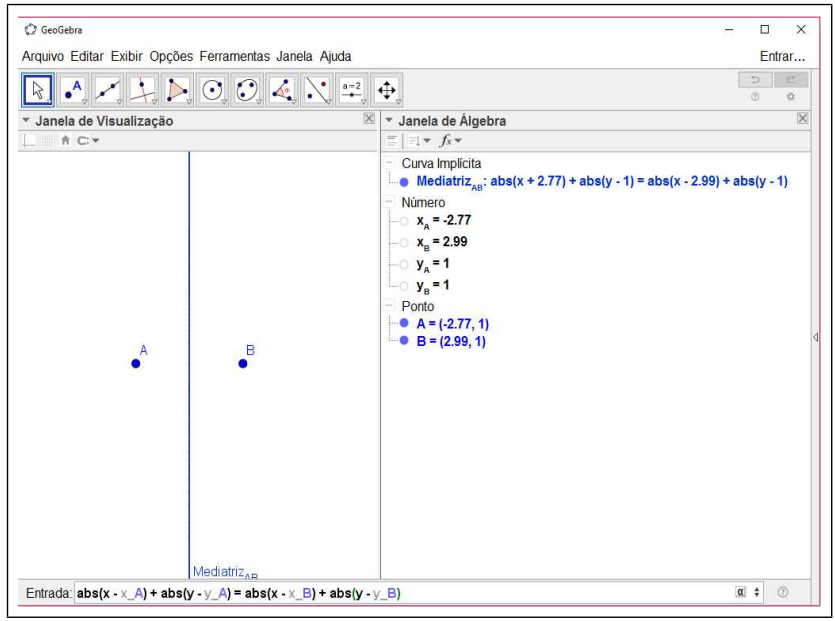

Figura 15. Táxi-Mediatriz no GeoGebra. 
Note que, dependendo da posição relativa entre os pontos $A$ e $B$, a táxi-mediatriz entre $A$ e $B$ pode ou não ser igual à mediatriz na métrica Euclidiana. Na Figura 15 a táximediatriz coincide com a mediatriz na métrica Euclidiana, mas na Figura 16, a táxi-mediatriz é formada por um segmento de reta unido a duas semirretas. A representação específica dependerá da inclinação $m$ entre os pontos $A$ e $B$.

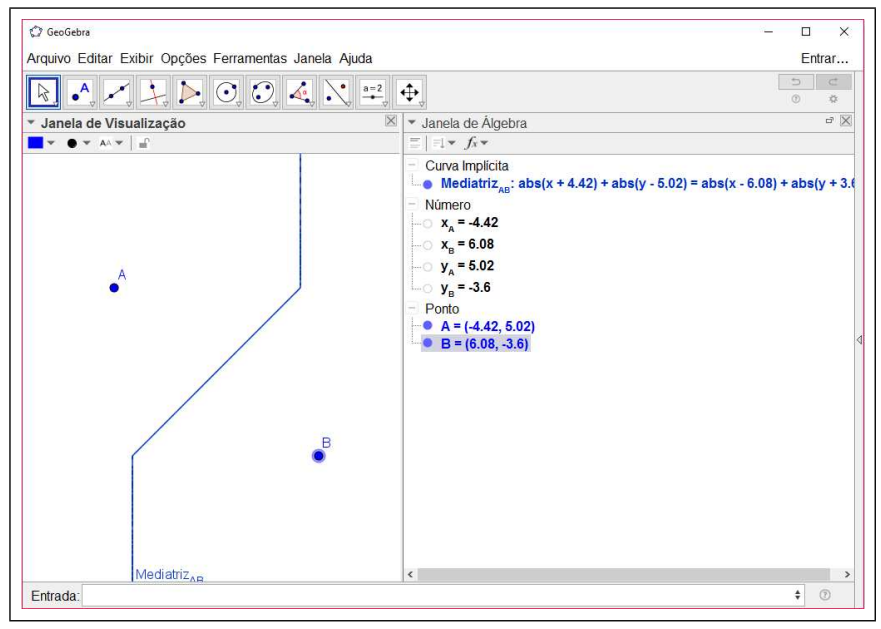

Figura 16. Táxi-Mediatriz no GeoGebra.

Usando diretamente a expressão (3) para gerar a representação da táxi-mediatriz no caso em que esta inclinação é igual a 1 ou -1 o resultado no GeoGebra pode parecer confuso, como sugerem as Figuras 17 e 18.

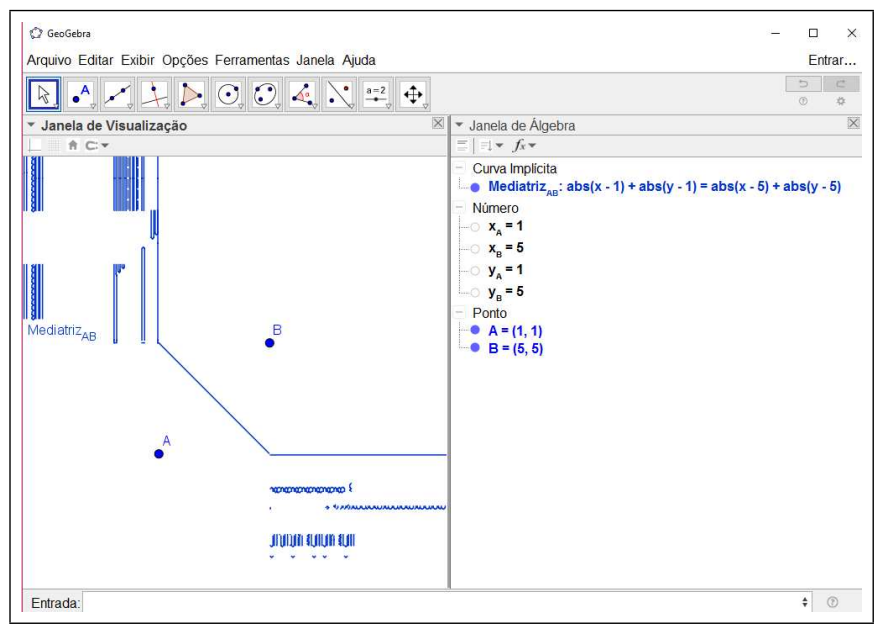

Figura 17. Táxi-Mediatriz em GeoGebra para $m=1$. 
Revista Ciências Exatas e Naturais, Vol.19, nº.2, Jul/Dez, 2017

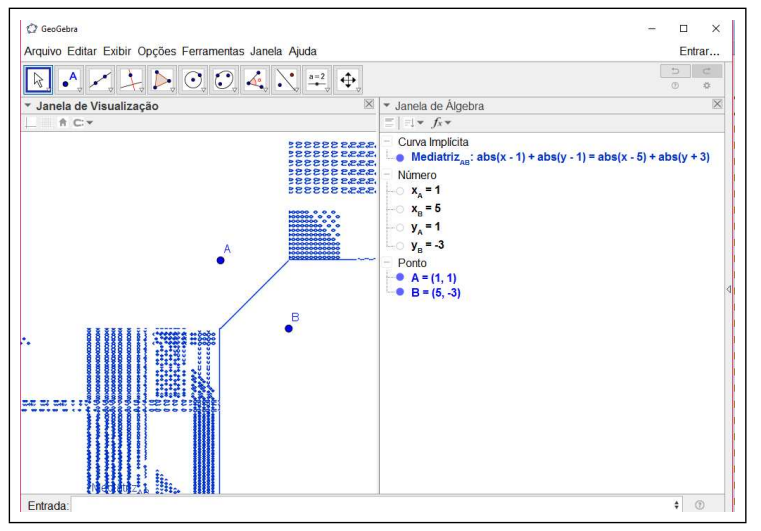

Figura 18. Táxi-Mediatriz no GeoGebra para $m=-1$.

Sem nenhum resultado teórico adicional, as representações apresentadas nas Figuras 17 e 18 são no mínimo confusas e podem até parecer fruto de algum erro. A implementação direta e simples da equação da táxi-mediatriz no GeoGebra, não permite identificar adequadamente o que acontece com a táxi-mediatriz quando $m=1$ ou $m=-1$, como pode ser verificado na construção dinâmica disponível em https://ggbm.at/RVCjwEjB. A seguir, serão apresentados os resultados teóricos que estabelecem adequadamente as caraterísticas da táxi-mediatriz.

Proposição 9 Dados dois pontos $A=\left(x_{a}, y_{a}\right)$ e $B=\left(x_{b}, y_{b}\right)$, seja $m$ a inclinação do segmento $\overline{A B}$. Se $m=0$ então a táxi-mediatriz entre $A$ e $B$ será a reta $x=\frac{x_{a}+x_{b}}{2}$. Se o segmento $\overline{A B}$ é vertical então $m$ é indefinida e a táxi-mediatriz será a reta $y=\frac{y_{a}+y_{b}}{2}$.

Note que neste caso a táxi-mediatriz coincide com a mediatriz na métrica Euclidiana. A Figura 19 ilustra este fato considerando dois pontos $A$ e $B$ e as distâncias entre os pontos $P_{1}$ e $P_{2}$ sobre a táxi-mediatriz e os pontos $A$ e $B$.

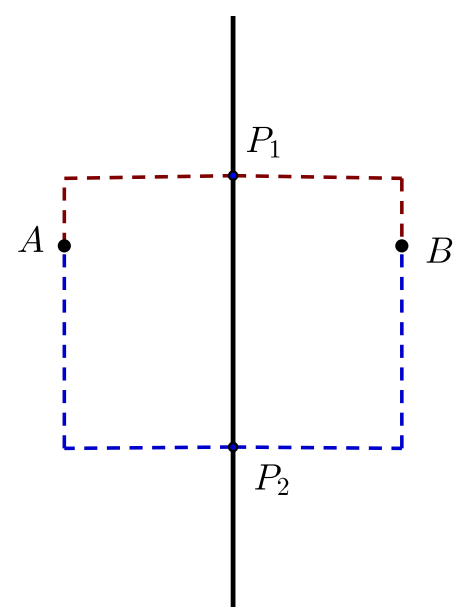

Figura 19. Táxi-mediatriz quando $m=0$. 


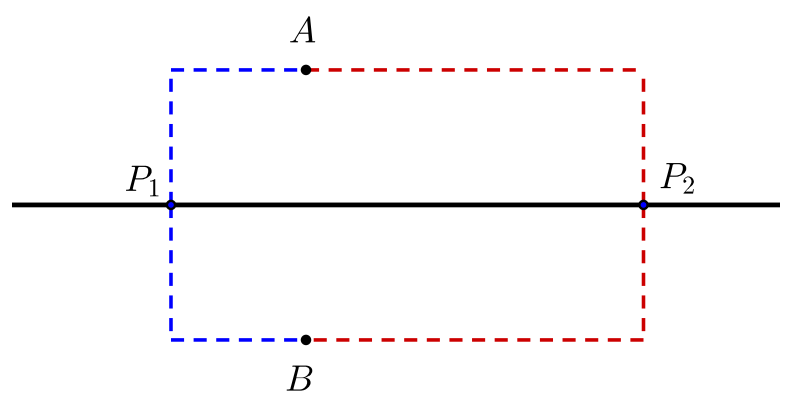

Figura 20. Táxi-mediatriz quando $m$ é indefinido.

O seguinte resultado considera os casos em que $|m| \neq 1$ e $m \neq 0$. As Figuras 21 e 22 representam as típicas táxi-mediatrizes para $m>1$ e $0<m<1$. Para um desenvolvimento mais construtivo deste resultado veja [6].

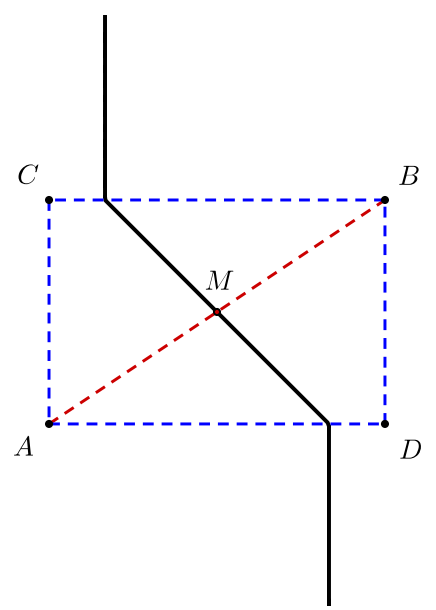

Figura 21. Táxi-mediatriz para $0<m<1$.

Proposição 10 Dados dois pontos $A=\left(x_{a}, y_{a}\right)$ e $B=\left(x_{b}, y_{b}\right)$, seja $m$ a inclinação do segmento $\overline{A B}$.

- Se $0<m<1$ então a táxi-mediatriz entre $A$ e $B$ é formada pelo segmento de reta entre os pontos $\left(x_{a}+\frac{\left(x_{b}-x_{a}\right)(1-m)}{2}, y_{b}\right)$ e $\left(x_{a}-\frac{\left(x_{b}-x_{a}\right)(1-m)}{2}, y_{a}\right)$, a parte da reta $x=x_{a}+\frac{\left(x_{b}-x_{a}\right)(1-m)}{2}$ correspondente $a y \geq y_{b}$ e a parte da reta $x=x_{b}-\frac{\left(x_{b}-x_{a}\right)(1-m)}{2}$ correspondente a $y \leq y_{a}$.

- Se $1<m$ então a táxi-mediatriz entre $A$ e $B$ é formada pelo segmento de reta entre os pontos $\left(x_{a}, y_{b}-\frac{\left(x_{b}-x_{a}\right)(m-1)}{2}\right) e\left(x_{b}, y_{a}+\frac{\left(x_{b}-x_{a}\right)(m-1)}{2}\right)$, a parte da reta 
Revista Ciências Exatas e Naturais, Vol.19, nº .2, Jul/Dez, 2017

$y=y_{a}+\frac{\left(x_{b}-x_{a}\right)(m-1)}{2}$ correspondente $a x \geq x_{b}$ e a parte da reta $y=y_{b}-\frac{\left(x_{b}-x_{a}\right)(m-1)}{2}$ correspondente a $x \leq x_{a}$.

- Se $-1<m<0$ então a táxi-mediatriz entre $A$ e $B$ é formada pelo segmento de reta entre os pontos $\left(x_{a}+\frac{\left(x_{b}-x_{a}\right)(1+m)}{2}, y_{b}\right)$ e $\left(x_{a}-\frac{\left(x_{b}-x_{a}\right)(1+m)}{2}, y_{a}\right)$, a parte da reta $x=x_{a}+\frac{\left(x_{b}-x_{a}\right)(1+m)}{2}$ correspondente $a y \leq y_{b}$ e a parte da reta $x=x_{b}-\frac{\left(x_{b}-x_{a}\right)(1+m)}{2}$ correspondente a $y \geq y_{a}$.

- Se $m<-1$ então a táxi-mediatriz entre $A$ e $B$ é formada pelo segmento de reta entre os pontos $\left(x_{a}, y_{b}+\frac{\left(x_{b}-x_{a}\right)(-m-1)}{2}\right) e\left(x_{b}, y_{a}-\frac{\left(x_{b}-x_{a}\right)(-m-1)}{2}\right)$, a parte da reta $y=$ $y_{a}-\frac{\left(x_{b}-x_{a}\right)(-m-1)}{2}$ correspondente $a x \geq x_{b}$ e a parte da reta $y=y_{b}+\frac{\left(x_{b}-x_{a}\right)(-m-1)}{2}$ correspondente $a x \leq x_{a}$.

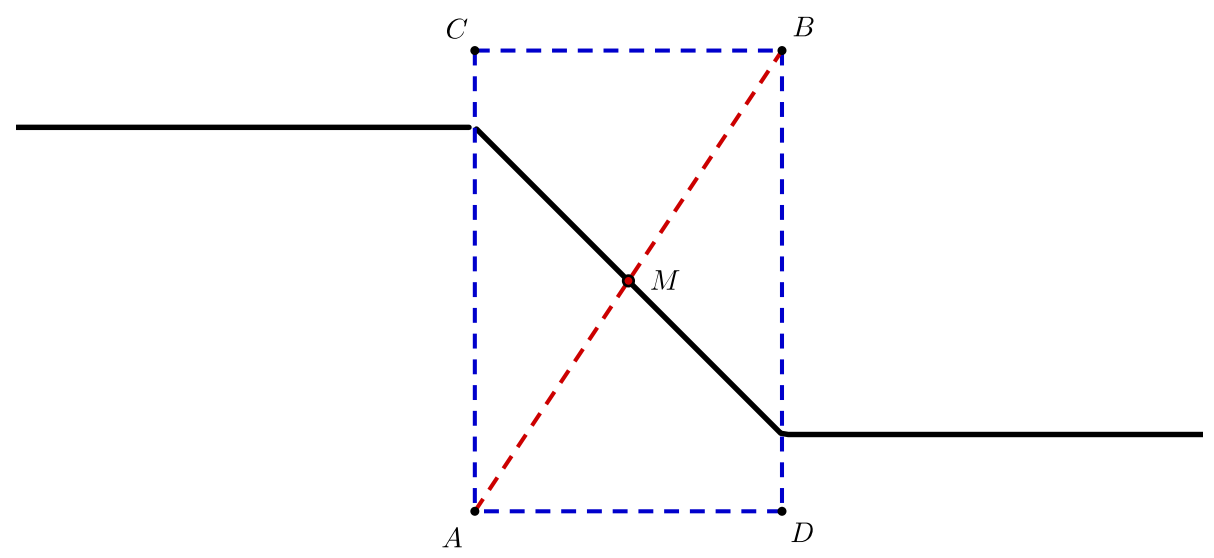

Figura 22. Táxi-mediatriz para $m>1$.

A proposição seguinte considera o caso em que $|m|=1$. Nesta situação a táxi-mediatriz não é formada apenas por retas ou segmentos de reta, sendo formada por um segmento de reta unido a dois quadrantes do plano como é ilustrado nas Figuras 23 e 24

Proposição 11 A táxi-mediatriz de um segmento com inclinação $|m|=1$ é formada pela união de um segmento de reta e dois quadrantes conforme os dois casos a seguir:

- Se $m=1$ a táxi-mediatriz é formada pelos quadrantes $\left(x \leq x_{a}, y \geq y_{b}\right) e\left(x \geq x_{b}\right.$, $\left.y \leq y_{a}\right)$, e pelo segmento da reta $y=\frac{x_{a}+y_{a}+x_{b}+y_{b}}{2}-x$ para $x_{a}<x<x_{b}$.

- Se $m=-1$ a táxi-mediatriz é formada pelos quadrantes $\left(x \leq x_{a}, y \leq y_{b}\right)$ e ( $x \geq x_{b}$, $\left.y \geq y_{a}\right)$, e pelo segmento da reta $y=\frac{-x_{a}+y_{a}-x_{b}+y_{b}}{2}+x$ para $x_{a}<x<x_{b}$.

Com os conceitos anteriores sobre a táxi-mediatriz, é possível construir uma ilustração mais completa no GeoGebra, como por exemplo a disponível em https://ggbm.at/aAwc9pdJ 1 .

${ }^{1}$ Autoria de Marco Antônio Manneta 


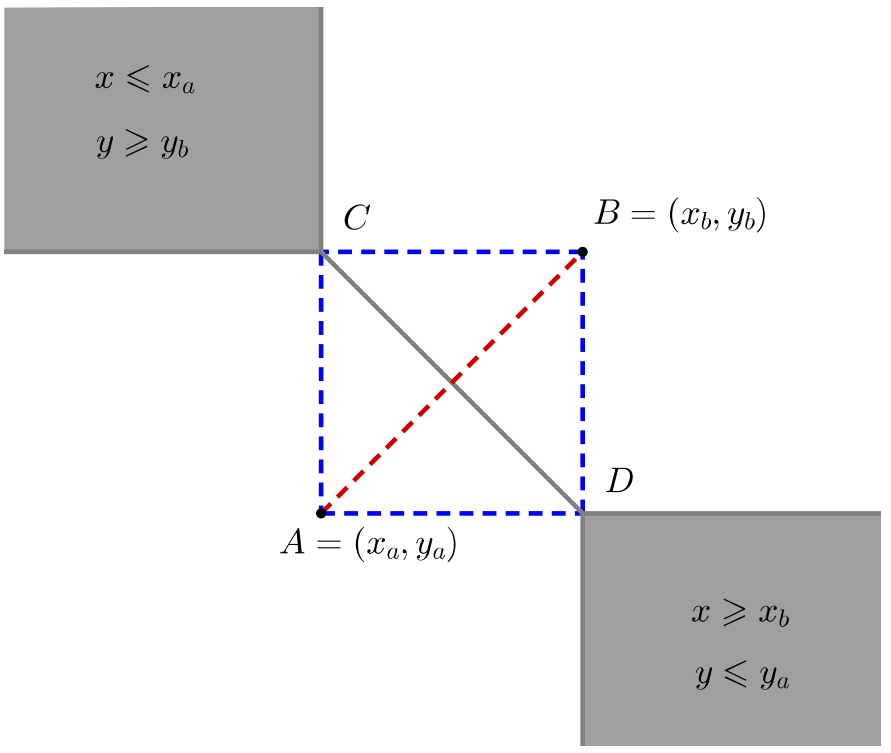

Figura 23. Táxi-mediatriz para $m=1$.

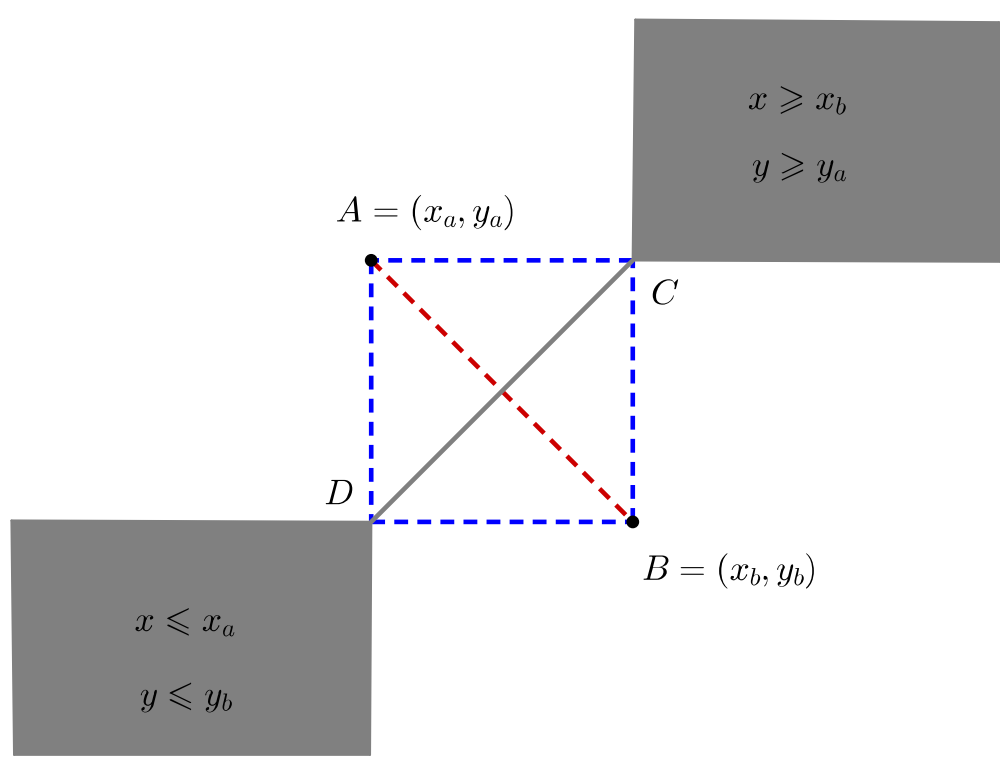

Figura 24. Táxi-mediatriz para $m=-1$. 
Revista Ciências Exatas e Naturais, Vol.19, nº.2, Jul/Dez, 2017

\section{Representação do diagrama de Voronoi na Métrica do Táxi}

Nesta seção será apresentado um procedimento para obter uma representação do diagrama de Voronoi na métrica do táxi. A ideia é a seguinte: Inicialmente considere para cada ponto do conjunto $P=\left\{p_{1}, p_{2}, \cdots, p_{n}\right\}$ uma táxi-circunferência de centro em $p_{i}$ e raio $r$.

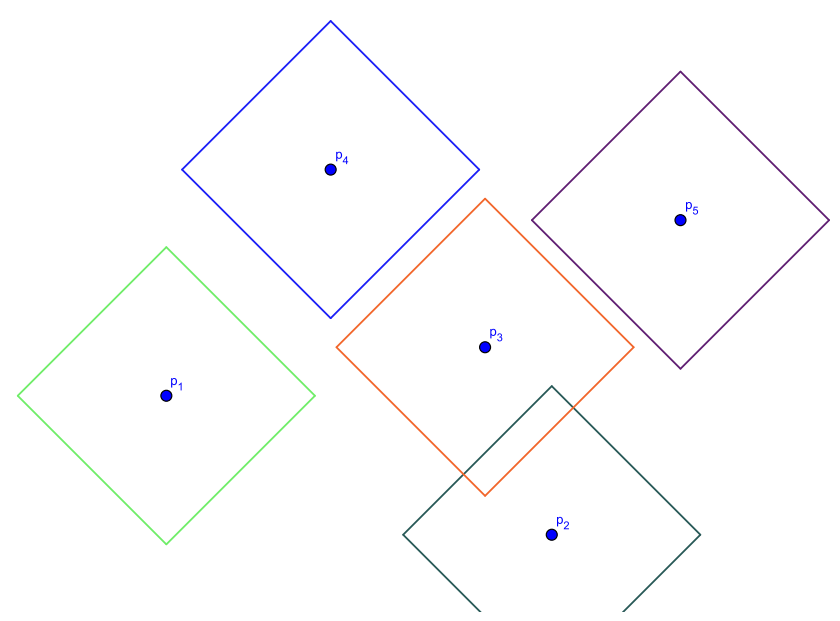

Figura 25. Táxi-Circunferências de centro em $p_{i}$ e raio $r$.

Considere também que o raio $r$ varia de forma contínua sobre o intervalo $[0, \infty)$. Quando $r=0$, o único ponto sobre a táxi-circunferência é o próprio ponto $p_{i}$, mas quando $r$ vai aumentando, qualquer ponto $p$ do plano estará em algum momento sobre a circunferência correspondente ao ponto $p_{i}$.

A primeira vez que $p$ estiver sobre a táxi-circunferência de centro em $p_{i}$, para algum $i$, isto seria equivalente a dizer que $p$ está mais próximo ou pelo menos a mesma distância de $p_{i}$ do que de qualquer outro ponto $p_{j}$, ou seja, $p$ pertence à célula de Voronoi $V\left(p_{i}\right)$ se é atingido primeiro pela circunferência correspondente a $p_{i}$. Por outro lado, se fosse possível que $r$ toma-se como valor inicial $\infty$ e fosse decrescendo até 0 , então todos os pontos estariam em algum momento sobre cada táxi-circunferências, mas só a última vez que um ponto $p$ fosse tocado por uma táxi-circunferência com centro em algum $p_{i}$, isto significaria que $p$ seria um elemento da célula associada ao ponto $p_{i}$.

É possível emular esta ideia no GeoGebra com o uso de cores dinâmicas e utilizando a opção de rastro para as táxi-circunferências. É claro que não é possível inicializar $r$ no valor $\infty$ mas para uma escolha inicial suficientemente grande, é possível obter uma representação aproximada do diagrama de Voronoi. 


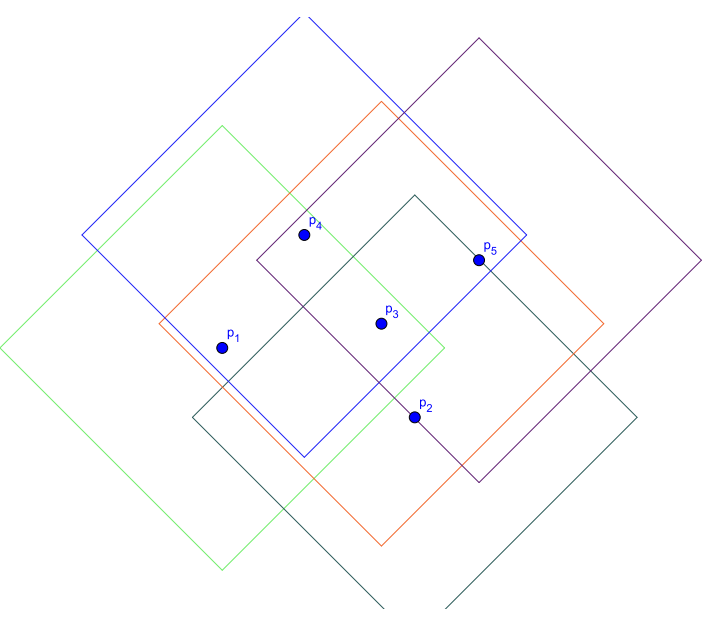

Figura 26. Representação do Diagrama de Voronoi na métrica do Táxi

No exemplo ilustrado na Figura 26 foi considerado $r$ com valor inicial 13. A Figura 27 mostra a situação quando quando $r=4$, e é possível notar como o rastro deixado pelas táxicircunferências vai colorindo diversos pontos do plano e as células correspondentes a cada ponto começam a ser esboçadas. Um mesmo ponto pode ter diferentes cores em diferentes momentos, mas a cor deixada pela última circunferência que o atinge, indicará a célula de Voronoi a qual pertence.

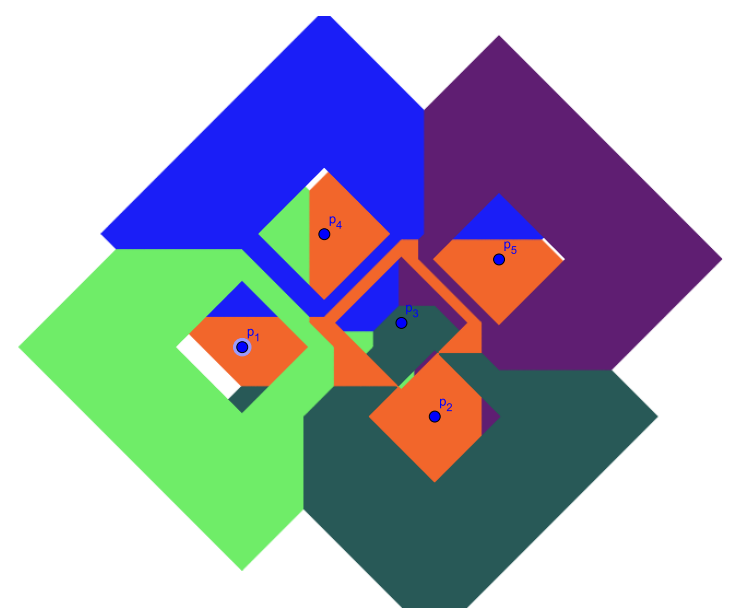

Figura 27. Representação do Diagrama de Voronoi na métrica do Táxi.

Na Figura 28 aparece o resultado final deste processo: uma representação aproximada do diagrama de Voronoi na métrica do táxi para $P=\left\{p_{1}, p_{2}, p_{3}, p_{4}, p_{5}\right\}$. 


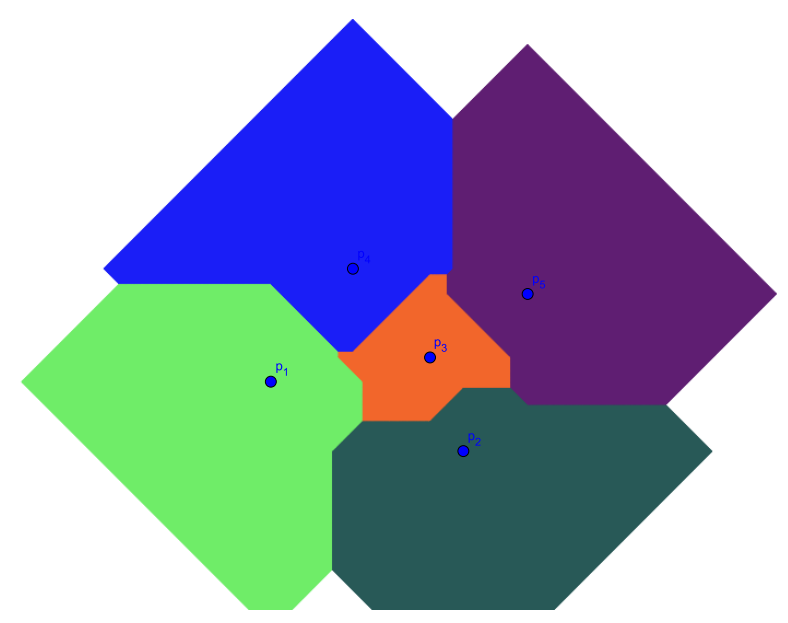

Figura 28. Representação do Diagrama de Voronoi na métrica do Táxi.

A partir da Figura 28, é possível ver que no caso da métrica do táxi, as células não são necessariamente regiões convexas. A Figura 28 parece sugerir também que as células ilimitadas correspondem a pontos $p_{i}$ na fronteira do fecho convexo de $P$, mas como é ilustrado na Figura 29, esto não é necessariamente verdade. O procedimento descrito anteriormente pode ser explorado na animação disponível em https://ggbm.at/US6vBbV4.

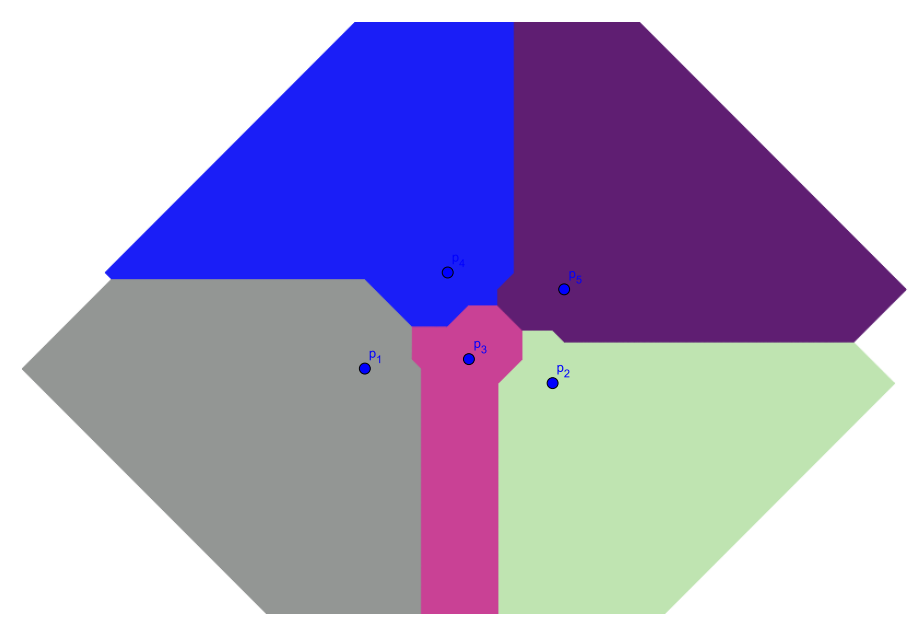

Figura 29. Célulás ilimitadas não correspondem a pontos na fronteira do fecho convexo.

\section{Comentários Finais}

Ao longo do trabalho foi descrita a utilização do GeoGebra, tanto para ilustrar resultados teóricos relacionados ao diagrama de Voronoi na métrica euclidiana, como para a exploração dos conceitos de táxi-circunferência e táxi-mediatriz. Estes resultados e conceitos estão baseados em ideias simples, mas nem sempre é imediata a sua compreensão. O uso do GeoGebra permite aprimorar a compreensão destes conceitos. Foi ilustrado também, que uma implementação direta no GeoGebra da equação da táxi-mediatriz, poderia levar a confusões sem 
SANTOS, P. S. e BAEZ-SANCHEZ, A. D.

a adequada fundamentação teórica. Foi descrito também como GeoGebra pode ser usado para produzir uma representação do diagrama de Voronoi na métrica do táxi. É importante destacar que este procedimento de representação pode ser estendido a outras métricas, se há uma construção adequada da circunferência correspondente. Finalmente, queremos destacar que as ligações entre as ideias aqui discutidas e alguns conceitos considerados no ensino médio, permitem ao professor de Matemática estabelecer situações-problemas interessantes, com aplicações diretas de conceitos geométricos e que quando desenvolvidas com ajuda de ferramentas computacionais como GeoGebra, podem estimular o processo de aprendizado.

\section{Referências}

[1] OKABE, A.; BOOTS, B.; SUGIHARA, K.; CHIU, S. N. Spatial Tessellations: Concepts and Applications of Voronoi Diagrams. Wiley Series in Probability and Statistics, 2009 .

[2] BERG, M. Computational Geometry: Algorithms and Applications. Springer, 2008.

[3] FigueiredO, L. H.; CARVAlhO, P. C. P. Notas de Geometria Computacional. IMPA, 2009.

[4] LIEBLING, T. M.; POURNIN, L. Voronoi Diagrams and Delaunay Triangulations: Ubiquitous Siamese Twins. Documenta Mathematica, Extra Volume ISMP, p.419-431, 2012 .

[5] JOHNSON, S. O mapa fantasma: Como a luta de dois homens contra a cólera mudou o destino de nossas metrópoles. Zahar, 2008.

[6] SANTOS, P. S. Diagrama de Voronoi: Uma exploração nas distâncias Euclidiana e do táxi. Dissertação de Mestrado, Mestrado Profissional em Matemática em Rede Nacional PROFMAT, 2016.

[7] KRAUSE, E. F. Taxicab Geometry: An Adventure in Non-Euclidean Geometry. Dover Publications, 1986.

[8] WANDERLEY A. J. M.; CARNEIRO J. P. Q. e WAGNER E. Como melhorar a vida de um casal usando geometria não-euclidiana. Revista do Professor de Matemática, v.50, p.23-30, 2001. 